\title{
ESTIMATIVA DE BEM-ESTAR DE FRANGO DE CORTE EM FUNÇÃO DA CONCENTRAÇÃO DE AMÔNIA E GRAU DE LUMINOSIDADE NO GALPÃO DE PRODUÇÃO
}

\author{
ADRIANA N. OWADA ${ }^{1}$, IRENILZA DE A. NÄ̈̈̈², DANIELLA J. DE MOURA ${ }^{3}$, \\ MARTA DOS S. BARACHO
}

\begin{abstract}
RESUMO: O Brasil é um importante país exportador de carne de frango, e grande parte de seu destino são países com restrições específicas de alojamento, relacionadas ao bem-estar das aves. Um dos poluentes aéreos freqüentemente encontrados em altas concentrações nos aviários, principalmente em ambientes fechados, é a amônia. Existem evidências de que o bem-estar de frangos de corte pode ser comprometido pela exposição contínua a esse poluente dentro dos galpões de alojamento. Este trabalho visou a estimar o bem-estar para frangos de corte alojados, a partir de atributos específicos do ambiente térmico e da densidade de aves, em função da concentração de amônia e luminosidade no ambiente de alojamento, utilizando a Teoria dos Conjuntos Fuzzy. Os resultados mostraram que o melhor valor de bem-estar (0,89 na escala: 0-1), aproximadamente $90 \%$ do ideal, foi encontrado nas condições que associam o ambiente térmico ideal, com densidade entre 13-15 aves $\mathrm{m}^{-2}$, com valores de concentração de amônia no ambiente abaixo de $5 \mathrm{ppm}$, e com luminosidade ambiente próxima de 1 lx. Utilizando o método preditivo, foi possível estimar o bem-estar de frangos de corte com relação à concentração de amônia no galpão e a sua luminosidade.
\end{abstract}

PALAVRAS-CHAVE: qualidade de ar, ambiente de alojamento, lógica Fuzzy.

\section{ESTIMATING BROILER WELFARE AS FUNCTION OF AMMONIA CONCENTRATION AND LIGHT LEVEL INSIDE HOUSING}

\begin{abstract}
Brazil is an important poultry meat export country, and large parts of its destination are countries with specific rearing restrictions related to broiler's welfare. One of the aerial pollutants mostly found in high concentrations in closed poultry housing environment is ammonia. There are evidences that broilers welfare may be compromised by the continuous exposition to this pollutant in rearing housing. This research aimed to estimate broilers welfare reared under specific thermal environmental attributes and bird's density, as function of the ammonia concentration and light intensity inside the housing environment using the Fuzzy Theory. Results showed that the best welfare value (0.89 in the scale: $0-1)$ approximately $90 \%$ of the ideal was found in the conditions that associated the ideal thermal environment, with bird's density between 13-15 birds $\mathrm{m}^{-2}$, with values of the ammonia concentration in the environment below $5 \mathrm{ppm}$, and light intensity near $1 \mathrm{~lx}$. Using the predictive method it was possible to estimate broilers welfare with relation to the ammonia concentration and light intensity in the housing.
\end{abstract}

KEYWORDS: air quality, broiler environment, fuzzy logic.

\footnotetext{
${ }^{1}$ Graduando de Engenharia Agrícola, FEAGRI-UNICAMP, Campinas - SP.

${ }^{2}$ Eng ${ }^{\mathrm{a}}$ Civil, FEAGRI-UNICAMP, Campinas - SP, irenilza@ agr.unicamp.br

${ }^{3}$ Eng $^{\mathrm{a}}$ Agrônoma, FEAGRI-UNICAMP, Campinas - SP, daniella.moura@agr.unicamp.br

${ }^{4}$ Bióloga, FEAGRI-UNICAMP, Campinas - SP, baracho@agr.unicamp.br

Recebido pelo Conselho Editorial em: 31-8-2006

Aprovado pelo Conselho Editorial em: 9-8-2007
} 


\section{INTRODUÇÃO}

A produção brasileira de carne de frango, em 2006, atingiu cerca de nove milhões de toneladas, crescimento de $4,1 \%$ acima da produção de 2005 . Além de movimentar uma receita anual de cerca de 30 milhões de dólares, a cadeia de produção de frangos de corte cria aproximadamente cinco milhões de empregos fixos e representa em torno de $6 \%$ do comércio exterior brasileiro, com um total de exportação da ordem de três milhões de toneladas, incluindo produtos in natura, industrializados e cortes (UBA, 2006). Dentre os destinos da carne de frango brasileira, encontram-se países da União Européia (14\%) e Japão (18\%), que possuem legislação restrita de condições de alojamento com relação ao bem-estar das aves.

No Brasil, a grande maioria dos galpões de frangos de corte que se destinam à exportação, provenientes de grandes integradoras, se encontra dentro de uma concepção similar de alojamento, tendo largura de 12 a $14 \mathrm{~m}$, comprimento de 70 a $100 \mathrm{~m}$ e pé-direito de cerca de $3 \mathrm{~m}$, com orientação solar leste-oeste. Dependendo da região, o sistema de túnel adaptado é utilizado, com cortinas laterais e sistemas de ventiladores e nebulizadores dispostos ao longo do galpão convencional. Diferentes técnicas de manejo dos ambientes térmicos e aéreos na avicultura podem contribuir efetivamente para a conquista da competitividade no mercado, quando associadas ao melhoramento genético, ao controle sanitário e à eficiência de produção das aves, o que resulta na melhoria da conversão alimentar e da taxa de crescimento diário, e que refletem positivamente no custo final do produto. Cada um desses fatores tem sua importância, sendo decisivo considerá-los de forma interdisciplinar (LIN et al., 2006).

A visão da sociedade com relação ao bem-estar animal está mudando, e isso tem ocorrido, principalmente, devido à rápida urbanização durante o último meio século, que, combinada com o aumento do poder aquisitivo, demanda ações específicas com relação ao ambiente e às condições dos criatórios dos animais alojados para consumo; dentre essas, destaca-se o bem-estar animal (EDWARDS, 2004; NÄÄS, 2005; MOURA et al., 2006). Ainda é universalmente aceita como medida de bem-estar animal, segundo DAWKINS (2003), a sua saúde física. Entretanto, o que ainda é considerado controverso é se somente essa medida seria suficiente, já que indicadores fisiológicos de bem-estar podem, eventualmente, ser uma resposta natural a atividades ou excitações naturais do animal, ao invés de indicar, especificamente, o seu bem-estar. Recente literatura sobre as questões de bem-estar na produção de aves (MARTRENCHAR et al., 1997; DAWKINS, 2003; EDWARDS, 2004; MOURA et al, 2006; BESSEI, 2006) indica que os temas relacionados à ambiência térmica e aérea, bem como à quantidade e intensidade de luz dos galpões, são abordados nas pesquisas isoladamente, sendo, entretanto, mais influentes na resposta das aves quando os extremos ocorrem simultaneamente.

Um dos poluentes aéreos freqüentemente encontrados em altas concentrações nos aviários, principalmente em ambientes fechados, é a amônia. Existem evidências de que a saúde animal pode ser comprometida pela exposição contínua a esse poluente, por meio de doenças respiratórias causadas por agentes oportunistas (SAINSBURY, 1981; HELLICKSON \& WALKER, 1983; HINZ \& LINKE, 1998). As aves submetidas a altas concentrações de amônia no galpão apresentam perdas produtivas e maior mortalidade (CAVENY \& QUARLES, 1978; REECE et al., 1980; CONCEIÇÃO et al., 1989; DONHAM et al., 2002).

No Brasil, não existem limites legais para a exposição de aves à amônia, entretanto exportadores de carne de frango adotam o limite de exposição constante máximo de $20 \mathrm{ppm}$, quando as concentrações de amônia em sistemas de criação intensiva fechados podem apresentar, na última semana de produção, valores de até 50 ppm (MIRAGLIOTTA, 2000; JONES et al., 2005).

Os conceitos de medida de qualidade do ar variam entre países, sendo influenciados pelas condições de alojamento das aves. Para HELLICKSON \& WALKER (1983), os fatores que intervêm na concentração de amônia nos aviários são: espécie, temperatura e taxa de ventilação. Já HINZ \& LINKE (1998) apontam que a distribuição dos poluentes aéreos nas instalações depende 
de sua tipologia, além do fluxo de ar e do seu padrão de circulação. MIRAGLIOTTA (2000), comparando os níveis de amônia em galpões de frango de corte em sistemas de ventilação e densidade diferenciados, mostrou que o sistema de ventilação tipo túnel removeu os gases gerados dentro das instalações que estavam com densidade de 18 aves $\mathrm{m}^{-2}$, garantindo qualidade de ar adequada, sendo mais eficiente do que o sistema de ventilação convencional, em instalações com densidade de 13-15 aves $\mathrm{m}^{-2}$.

Dentre as estratégias mais importantes para prevenir a perda de produtividade devido ao estresse calórico, está o controle da luminosidade em aviários (LIN et al., 2006), cujos autores reportam que frangos de corte expostos a baixa luminosidade $(<5 \mathrm{~lx})$ produzem menos calor sensível na quarta e quinta semanas. JONES et al. (2005), estudando o efeito simultâneo da exposição de frangos a diferentes concentrações de amônia e intensidades de luz, encontraram que as aves apresentaram comportamento aversivo ao acréscimo de concentração de amônia (acima de $10 \mathrm{ppm})$ no ambiente, diretamente proporcional ao acréscimo da intensidade luminosa. BESSEI (2006), revisando pontos críticos de bem-estar na produção de frangos de corte, aponta como importante o controle (6-10 lx) do regime e da intensidade de luz em aviários, por influenciar diretamente na atividade motora e possível exaustão das aves, além do aparecimento de anormalidades locomotoras.

Para a estimativa de situações com alto grau de aleatoriedade, como o bem-estar, baseada em análise empírica de medidas fisiológicas, pesquisas apontam o potencial do uso de critérios específicos em uma base de conhecimento prévio, utilizando a Teoria dos Conjuntos Fuzzy, usada na área de sistemas de suporte à decisão, que se caracterizam pela incerteza existente entre as afirmações de "sim" e "não" (AMENDOLA et al., 2004). A Teoria dos Conjuntos Fuzzy é baseada na linguagem expressa por variáveis lingüísticas, que podem ser transformadas em valores, cujo princípio é o "Postulado da Possibilidade" (BELLMAN \& ZADETH, 1970). A estimativa de bemestar animal, considerando todas as possibilidades de associações críticas do ambiente térmico e aéreo, constitui-se em um problema onde a aplicação da Teoria dos Conjuntos Fuzzy pode apresentar-se promissora. OLIVEIRA et al. (2005) estimaram o conforto térmico para alojamento de aves poedeiras em produção, encontrando, para essas aves, a melhor situação de conforto térmico $(0,87$, em uma escala de $0-1)$, para a temperatura de bulbo seco de $22,8{ }^{\circ} \mathrm{C}$, e umidade relativa do ar de $78,1 \%$, que concordava com valores de termoneutralidade encontrados na literatura (CURTIS, 1983; ALBRIGHT, 1990).

Este trabalho teve como objetivo analisar a composição das variáveis ambientais independentes: concentração de amônia e luminosidade no galpão, que influenciam na variável dependente aqui denominada de bem-estar, em galpões de frangos de corte, respeitando o cenáriolimite dos atributos do alojamento, utilizando a Teoria dos Conjuntos Fuzzy.

\section{MATERIAL E MÉTODOS}

A estrutura básica de um sistema baseado em regras Fuzzy inclui quatro componentes principais: um fuzzificador, que traduz a informação de entrada em Conjuntos Fuzzy; uma base de conhecimento, que contém um conjunto de regras fuzzy (conhecido como base de regras) e um conjunto de funções de pertinência (conhecido como base de dados); um método de inferência, que é aplicado para obter uma saída fuzzy, e um defuzzificador, que traduz a saída por um valor numérico.

Os dados relativos à base de conhecimento, neste trabalho, foram organizados de maneira a, inicialmente, estabelecer o limite superior dos atributos da ambiência térmica, proposta pelo conhecimento encontrado na literatura (CURTIS, 1983; ALBRIGHT, 1990; AMENDOLA et al., 2004; OLIVEIRA et al., 2005), da concentração de amônia (WATHES et al., 1997; MIRAGLIOTTA, 2000; JONES et al., 2005) e da associação da concentração de amônia e luminosidade (JONES et al., 2005; BESSEI, 2006). Na Tabela 1, reúnem-se os valores máximos dos atributos propostos na literatura, indicando o limite superior da situação ideal de bem-estar no alojamento, com relação aos ambientes térmico e aéreo, para frangos de corte alojados da quinta 
até a sétima semana. Essas condições foram estipuladas como limítrofes para o bem-estar em aves baseadas no conhecimento publicado (DAWKINS, 2003; MOURA et al., 2006; BESSEI, 2006; LIN et al., 2006), com densidade de 13-15 aves $\mathrm{m}^{-2}$ de piso e considerando que as demais condições de nutrição, sanidade e movimentação de ar estivessem dentro das condições ideais de alojamento, de acordo com as características climáticas regionais.

TABELA 1. Cenário-limite de bem-estar de frangos de corte alojados em galpão de produção intensiva com relação à ambiência térmica e aérea.

\begin{tabular}{cc}
\hline Atributos do Ambiente de Alojamento & Limite Superior \\
\hline Temperatura de bulbo seco & $23,0-30,0\left({ }^{\circ} \mathrm{C}\right)$ \\
Umidade relativa & $78-80(\%)$ \\
Luminosidade & Baixa \\
Concentração de amônia & $<5$ ppm \\
\hline
\end{tabular}

$\mathrm{Na}$ Tabela 2, consta a formação da base de regras considerando o bem-estar de frango de corte influenciado pela concentração de amônia e pela luminosidade dentro do galpão. Nessa planilha, a variável lingüística concentração de amônia no galpão (ppm), associada às condições já consideradas na Tabela 1 , foi dividida em faixas de $(0-10 ; 11-15 ; 16-20 ; 21-30 ; 31-40)$, com o domínio no intervalo de [0,40], com as seguintes denominações: muito bom, bom, média, ruim e muito ruim, respectivamente. Todas as classificações foram formuladas baseados nos trabalhos de WATHES et al. (1997), MIRAGLIOTTA (2000), MOURA et al. (2006) e BESSEI (2006). Para a variável lingüística luminosidade (lx), foram estabelecidas faixas de $(0-9 ; 10-99 ; 100)$, com o domínio no intervalo [0,100], com as seguintes denominações: baixa, média e alta, respectivamente, segundo JONES et al. (2005) e LIN et al. (2006).

As variáveis independentes constituíram intervalos de concentração de amônia (ppm) e de luminosidade (lx), enquanto a variável dependente, denotada por bem-estar, considerava que os atributos do ambiente estavam dentro dos limites admissíveis (Tabela 1), para a densidade de aves entre 13-15 aves $\mathrm{m}^{-2}$ e constitui intervalos no domínio de [0,1], também classificados segundo o mesmo conceito para frangos de corte, pelos termos ruim, médio, bom e muito bom, com pesos de zero a um para cada valor lingüístico (JONES et al., 2005; BESSEI, 2006). O valor entre parênteses, que é o peso da informação, foi consignado por especialista.

A partir da base de regras, foi formulado um controlador de lógica fuzzy utilizando o método de Mandani, tendo sido selecionadas as funções trapezoidais, de acordo com LANZILLOTTI (1999), AMENDOLA et al. (2004) e OLIVEIRA et al. (2005), e o sistema computacional usado no processamento foi o MATLAB ${ }^{\circledR}(2003)$.

TABELA 2. Base de regras para bem-estar de frango de corte em função de concentração de amônia e da luminosidade dentro do alojamento.

\begin{tabular}{cccccc}
\hline \multirow{2}{*}{ Luminosidade } & \multicolumn{5}{c}{ Concentração de Amônia (ppm) } \\
\cline { 2 - 6 } & $0-10$ & $11-15$ & $16-20$ & $21-30$ & $31-40$ \\
\hline Alta & muito bom $(0,7)$ & muito bom $(0,6)$ & médio $(0,9)$ & médio $(0,8)$ & muito ruim $(0,8)$ \\
Média & muito bom $(0,8)$ & bom $(0,8)$ & médio $(0,8)$ & ruim $(0,5)$ & ruim $(0,9)$ \\
Baixa & muito bom $(1,0)$ & médio $(0,9)$ & médio $(0,7)$ & ruim $(0,6)$ & ruim $(1,0)$ \\
\hline
\end{tabular}

Obs: Número entre parênteses significa peso da afirmação.

\section{RESULTADOS E DISCUSSÃO}

Tomando por base as questões incertas, com relação aos limites do que é recomendado na literatura com relação às variáveis dependentes e mantendo constantes os atributos do ambiente térmico (temperatura de bulbo seco e umidade relativa) e uma densidade de alojamento entre 13 - 15 aves $\mathrm{m}^{-2}$, foi criado um cenário para a variável de bem-estar para frangos de corte 
num domínio $[0,1]$. O valor próximo do zero indicava a pior condição de bem-estar, enquanto aquele perto do 1 , a melhor.

O método de inferência Mandani combinou o grau de pertinência referente a cada um dos valores de entrada, por meio do operador mínimo, e agregou as regras por meio do operador máximo. A defuzzificação realizada pelo método do centro de gravidade (Figura 1) permitiu a possibilidade de simular distintas situações entre as variáveis de entrada e obter resposta otimizada da variável de saída, como também utilizaram AMENDOLA et al. (2004) e OLIVEIRA et al. (2005).

A resposta do sistema de inferência para a concentração de amônia dentro do galpão, mostrada na Figura 1, foi igual a zero, e luminosidade de 1 lx. Após a defuzzificação, encontra-se a melhor situação possível para o nível de bem-estar de frangos de corte, no valor de 0,89 (escala: 01), mostrando que esses valores de concentração de amônia e luminosidade no galpão geram condições de bem-estar aproximadamente $90 \%$ do ideal.

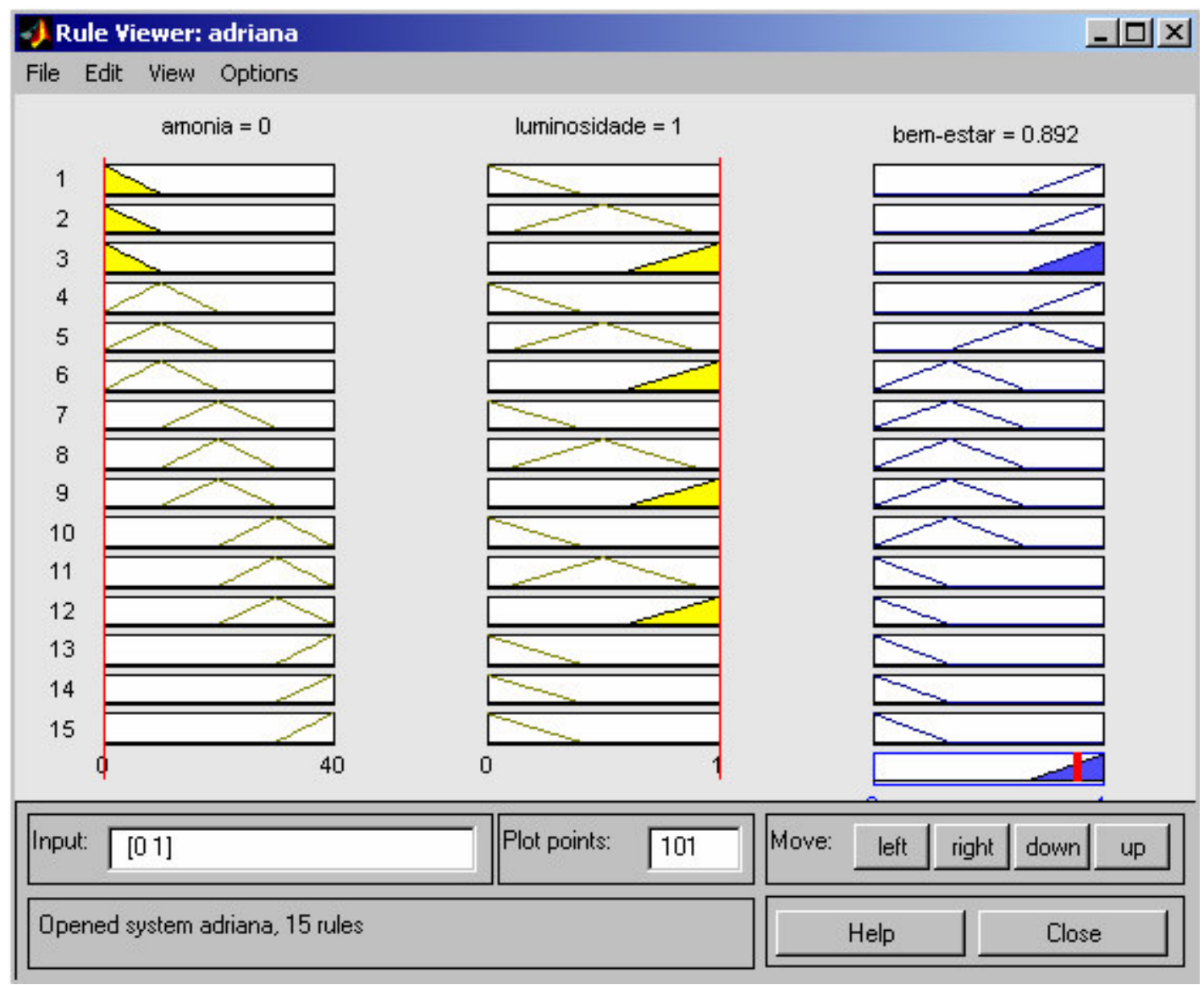

FIGURA 1. Base de regra com melhor situação de associação dos valores das variáveis dependentes (concentração de amônia e luminosidade do galpão), tendo como variável de saída o bem-estar das aves.

Na Figura 2, pode-se visualizar a variação não-linear do bem-estar, como função da concentração de amônia e luminosidade, que foi gerada a partir da base de regras. Dentro desse escopo, os melhores valores de bem-estar foram encontrados nas condições que associam o ambiente térmico ideal com valores de concentração de amônia no ambiente abaixo de 5 ppm, e com luminosidade ambiente próxima de 1 lx. A partir desses valores, fica nítida a aversão da ave àquela situação de ambiente, o que caracteriza ausência parcial ou total de bem-estar, de acordo com DAWKINS (2003) e EDWARDS (2004); portanto, seriam esses os limites de bem-estar ótimo para as condições de ambiência térmica prefixadas. 


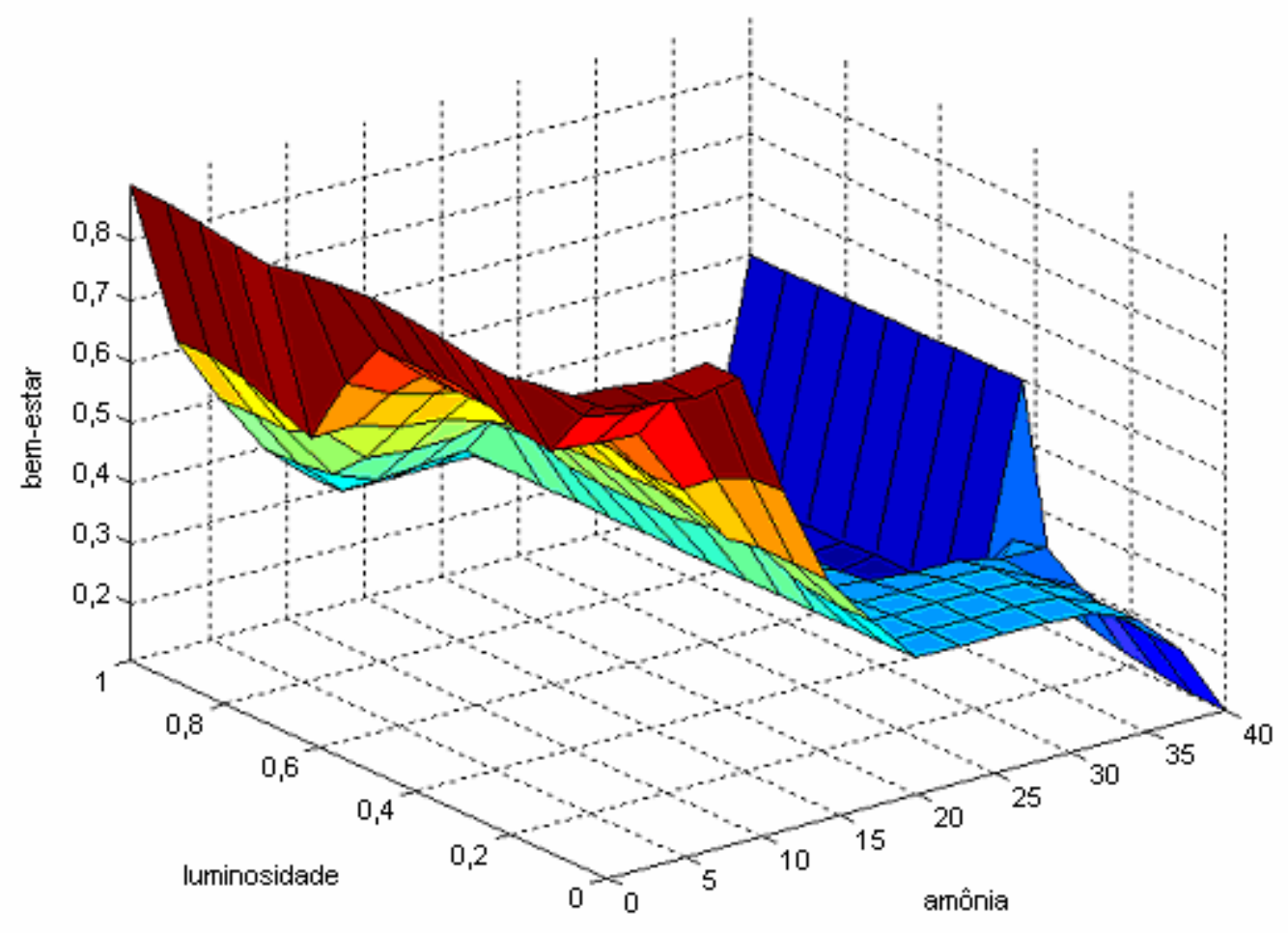

FIGURA 2. Estimativa de bem-estar para frango de corte em galpão de produção, considerando a condição ótima de concentração de amônia e a luminosidade do galpão.

No caso da concentração de amônia, na prática, valores abaixo de 5 ppm nos galpões de produção são encontrados raramente e, segundo WATHES et al. (1997), MIRAGLIOTTA (2000) e JONES et al. (2005), as concentrações de amônia podem chegar até $50 \mathrm{ppm}$ dentro das granjas, sendo considerado o normalmente encontrado em situações boas de alojamento, na ordem de 10 - 20 ppm, o que seria uma concentração média de amônia. Resultados obtidos por CAVENY \& QUARLES (1978) indicam que níveis de amônia de 25 e 50 ppm podem afetar o desempenho de frangos, por isso selecionar o limite de $20 \mathrm{ppm}$ estaria dentro do admissível. No tocante à luminosidade, a recomendação de BESSEI (2006) e LIN et al. (2006) é que seja baixo, entretanto, acima de zero, leva a que se estabeleça o valor ideal em torno de 1-5 lx, que seria o cenário de média luminosidade. Para essas condições, não se poderiam ter condições ótimas de bem-estar, mas viáveis, conforme apontam DAWKINS (2003) e BESSEI (2006).

Dessa forma, por meio da estimativa do bem-estar de frangos de corte, observou-se que os resultados obtidos no presente trabalho são menores do que aqueles preconizados por WATHES et al. (1997) e LIN et al. (2006), que indicam cenários com ambientes fechados e totalmente controlados, assemelhando-se às recomendações de BESSEI (2006).

\section{CONCLUSÔES}

Utilizando a Teoria dos Conjuntos Fuzzy, no ambiente de computação científica MATLAB® (2003), foi possível, no cenário de atributos de ambiente térmico e densidade de aves predeterminados, estimar o bem-estar de frangos de corte com relação à concentração de amônia e grau de luminosidade em galpão de produção.

\section{REFERÊNCIAS}

ALBRIGHT, L.D. Environmental control for animals and plants. St. Joseph: ASABE, 1990. 318 p. 
AMENDOLA, M.; CASTANHO, M.J.; NAAS, I.; SOUZA, A.L. Análise matemática de condições de conforto térmico para avicultura usando a teoria dos conjuntos Fuzzy. Biomatemática Brasil, Campinas, v.14, n.1, p.87-92, 2004.

BELLMAN, R.E.; ZADETH, L.A. Decision-making in a fuzzy environment. Management Science, Evanston, v.17, n.4, p.141-64, 1970.

BESSEI, W. Welfare of broilers. World's Poultry Science Journal, Beekbergen, v.62, n.3, p.45566, 2006.

CAVENY, D.D.; QUARLES, C.L. The effect of atmospheric ammonia stress on broiler performance and carcass quality. Poultry Science, Atlanta, v.57, n.3, p.1124-8, 1978.

CONCEIÇÃO, M.A.P.; HAZEL, E.J.; WATHES, C.M. Air hygiene in a pullet house: spatial homogeneity of aerial pollutants. British Poultry Science, Londres, v.30, n.4, p.765, 1989.

CURTIS, S.E. Environmental management in animal agriculture. Ames: The Iowa State University Press, 1983. 410 p.

DAWKINS, M.S. Behavior as a tool in the assessment of animal welfare. Zoology, Berlim, v.106, n.4, p.383-7, 2003.

DONHAM, K.J.; THORNE, P.S.; BREUER, G.M.; POWERS, W.; MARQUEZ, S.; REYNOLDS, S.J. Exposure limits related to air quality and risk assessment. Iowa concentrated animal feeding operation - air quality study. Ames: Environmental Health Sciences Research Center, University of Iowa, 2002. $12 \mathrm{p}$.

EDWARDS, J.D. The role of the veterinarian in animal welfare - a global perspective. Global Conference on Animal Welfare: an OIE Initiative - 2004. Disponível em: http://www.oie.int/eng/Welfare\%5F2004/home.htm. Acesso em: 16 out. 2005.

HELLICKSON, M.A.; WALKER, J.N. Ventilation of Agricultural Structures. St. Joseph: ASABE, 1983, 23 p. (Monograph, 6).

HINZ, T.; LINKE, S. A comprehensive experimental study of aerial pollutants in and emissions from livestock buildings. Part 1: Methods. Journal of Agricultural Engineering Research, Silsoe, v.70, n.1, p.111-9, 1998.

JONES, E.K.M.; WATHES, C.M.; WEBSTER, A.J.F. Avoidance of atmospheric ammonia by domestic fowl and the effect of early experience. Applied Animal Behaviour Science, Londres, v.90, n.3, p.293-308, 2005.

LANZILLOTTI, R.S. Análise sensorial sob enfoque da decisão Fuzzy. Revista de Nutrição, Campinas, v.12, n.2, p.145-9, 1999.

LIN, H.; JIAO, H.C.; BUYSE, J.; DECUYPERE, F. Strategies for preventing heat stress in poultry. World's Poultry Journal, Beekbergen, v.62, n.1, p.71-85, 2006.

MARTRENCHAR, A.; MORISSE, J.P.; HUONNIC, D.; COTTE, J.P. Influence of stocking density on some behavioural, physiological and productivity traits broiler. Veterinary Research, Les Ulis, v.28, n.5, p.473-80, 1997.

MATLAB®. The Language of Technical Computing. Natick: The Mathworks Inc. Versão 6.1. 2003. Disponível em: http://www.mathworks.com. Acesso em: 2 fev. 2004.

MIRAGLIOTTA, M.Y. Avaliação dos níveis de amônia em dois sistemas de produção de frangos de corte com ventilação e densidade diferenciados. 2000. 112 f. Dissertação (Mestrado em Construções Rurais e Ambiência) - Faculdade de Engenharia Agrícola, Universidade Estadual de Campinas, Campinas, 2000. 
MOURA, D.J.; NÄÄS, I. A.; PEREIRA, D.F.; SILVA, R.B.T.R.; CAMARGO, G.A. Animal welfare concepts and strategy for poultry production: a review. Revista Brasileira de Ciência Avícola, Campinas, v.8, n.1, p.137-48, 2006.

NÄÄS, I. A. Bem-estar na avicultura: fatos e mitos. Revista AveWorld, Campinas, v.10, ago./set., p.4-8, 2005.

OLIVEIRA, H.L.; AMENDOLA, M.; NÄÄS, I. A. Estimativa das condições de conforto térmico para avicultura de postura usando a teoria dos conjuntos fuzzy. Engenharia Agrícola, Jaboticabal, v.25, n.2, p.300-7, 2005.

REECE, F.N.; LOTT, B.D.; DEATON, J.W. Ammonia in the atmosphere during brooding affects performance of broiler chickens. Poultry Science, Atlanta, v.59, n.1, p.486-8, 1980.

SAINSBURY, D.W.B. Health problems in intensive animal production. In: CLARK, J.A.

Environmental aspects of housing for animal production. Londres: Butterworths, 1981. p.439-532.

UBA. União Brasileira de Avicultura. Disponível em:

http://www.uba.org.br/ubanews_files/relatorio_uba_06_07_baixa_1.pdf. Acesso em: 13 jul.2007.

WATHES, C.M.; HOLDEN, M.R.; SNEATH, R.W.; WHITE, R.P.; PHILLIPS, V.R.

Concentrations and emissions rates of aerial ammonia, nitrous oxide, methane, carbon dioxide, dust and endotoxin in UK broiler and layer houses. British Poultry Science, Londres, v.38, n.1, p.14-28, 1997. 